\title{
PIK3CA-mediated PI3-kinase signalling is essential for HPV-induced transformation in vitro
}

\author{
Florianne E Henken ${ }^{1}$, N Sanjib Banerjee ${ }^{2}$, Peter JF Snijders' ${ }^{1}$, Chris JLM Meijer', Johanna De-Castro Arce ${ }^{3}$, \\ Frank Rös $\left.\right|^{3}$, Thomas R Broker ${ }^{2}$, Louise T Chow ${ }^{2}$ and Renske DM Steenbergen ${ }^{*}$
}

\begin{abstract}
Background: High-risk human papillomavirus (hrHPV) infections are causally related to cervical cancer development. The additional (epi)genetic alterations driving malignant transformation of hrHPV-infected cells however, are not yet fully elucidated. In this study we experimentally assessed the role of the PI3-kinase pathway and its regulator PIK3CA, which is frequently altered in cervical cancer, in HPV-induced transformation.
\end{abstract}

Methods: Cervical carcinomas and ectocervical controls were assessed for PIK3CA mRNA and protein expression by quantitative RT-PCR and immunohistochemical staining, respectively. A longitudinal in vitro model system of hrHPVtransfected keratinocytes, representing the immortal and anchorage independent phenotype, was assayed for PI3kinase activation and function using chemical pathway inhibition i.e. LY294002 treatment, and PIK3CA RNA interference. Phenotypes examined included cellular viability, migration, anchorage independent growth and differentiation. mRNA expression of hTERT and HPV16 E6E7 were studied using quantitative RT-PCR and Northern blotting.

Results: Cervical carcinomas showed significant overexpression of PIK3CA compared to controls. During HPVinduced transformation in vitro, expression of the catalytic subunit PIK3CA as well as activation of downstream effector PKB/AKT progressively increased in parallel. Inhibition of PI3-kinase signalling in HPV16-transfected keratinocytes by chemical interference or siRNA-mediated silencing of PIK3CA resulted in a decreased phosphorylation of PKB/AKT. Moreover, blockage of PI3-kinase resulted in reduced cellular viability, migration, and anchorage independent growth. These properties were accompanied with a downregulation of HPV16E7 and hTERT mRNA expression. In organotypic raft cultures of HPV16- and HPV18-immortalized cells, phosphorylated PKB/ AKT was primarily seen in differentiated cells staining positive for cytokeratin 10 (CK10). Upon PI3-kinase signalling inhibition, there was a severe impairment in epithelial tissue development as well as a dramatic reduction in $\mathrm{p}$ PKB/AKT and CK10.

Conclusion: The present data indicate that activation of the PI3-kinase/PKB/AKT pathway through PIK3CA regulates various transformed phenotypes as well as growth and differentiation of HPV-immortalized cells and may therefore play a pivotal role in HPV-induced carcinogenesis.

\section{Background}

It has been well established that high-risk human papillomavirus (hrHPV) infections are causally related to cervical cancer development [1]. While the two most potent viral oncogenes E6 and E7 are necessary for the initiation and maintenance of cellular proliferation $[2,3]$, their expression is not sufficient for full transformation

\footnotetext{
* Correspondence: r.steenbergen@vumc.nl

'Department of Pathology, Unit of Molecular Pathology, VU University Medical Center, PO Box 7057, 1007 MB Amsterdam, The Netherlands Full list of author information is available at the end of the article
}

of epithelial cells. Hence, there are extensive efforts towards identifying additionally required host cell events. Chromosomal analysis has revealed that a gain of chromosome $3 q$ is the most common event in the development of cervical squamous cell carcinoma (SCC) [4-6]. In fact, a gain of chromosome 3q was found in all SCC previously analysed by microarray CGH [6]. Candidate oncogenes on chromosome $3 \mathrm{q}$ include the gene encoding $\mathrm{p} 110 \alpha$, the active subunit phosphatidylinositol 3-kinase catalytic alpha (PIK3CA) of class I PI3-kinase. Upon activation, PI3-kinase initiates events leading to

\section{Biomed Central}


phosphorylation of $\mathrm{PKB} / \mathrm{AKT}$, which affects additional downstream signalling proteins involved in survival and cell growth. Indeed, deregulation of the PI3-kinase pathway is common in many human malignancies [7]. In cervical carcinomas, an increased copy number of PIK3CA was positively correlated with an increase in phosphorylated $\mathrm{PKB} / \mathrm{AKT}$, one of the downstream effectors [8]. Additionally, the level of p-PKB/AKT expression increased proportional to the histopathological grade of (pre)malignant cervical diseases $[9,10]$. Although it has been found that HPV16E7 can activate $\mathrm{PKB} / \mathrm{AKT}$ in differentiating cells [10], the relevance of PI3-kinase signalling in the process of cervical cancer development following a transforming hrHPV infection remains to be experimentally explored. Moreover, no functional studies on the specific role of PIK3CA in cervical carcinogenesis have yet been performed.

Previously we have shown that in vitro transformation of primary keratinocytes mediated by full-length hrHPV was accompanied with a gain of chromosome $3 q$ in immortalized descendants [6]. This model system of HPV-transformed keratinocytes therefore provides interesting and useful source material to study the potential functional role of PI3-kinase for the various transformed phenotypes. In the present study we analysed PIK3CA expression in cervical squamous cell carcinomas. We also performed functional analyses of the contribution of PI3-kinase signalling, and specifically PIK3CA, to hrHPV-mediated transformation in vitro.

\section{Methods}

\section{Cell culture, LY294002 treatment and transfection}

Primary human foreskin keratinocytes, HPV16 and HPV18-immortalized keratinocyte cell lines (FK16A and FK18A) as well as HPV16E6E7 containing keratinocytes were cultured as described previously [11]. The latter cells were generated by transduction of primary human foreskin keratinocytes with the retroviral vector pLZRSneo containing HPV16E6E7, as described previously [12].

FK16A cells between passages 45 and 62 represented immortal and anchorage dependent cells and FK16A cells between passages 99 and 189 represented anchorage independent cells [11]. Prior to LY294002 (10 $\mu \mathrm{M}$ and $20 \mu \mathrm{M})$ (Cell Signaling Technology, Beverly, USA) or DMSO treatment, cells were starved overnight to ensure similar phosphorylation status. A pool of 4 siRNA sequences targeting PIK3CA (cat\#L-003018-000005, Dharmacon, Lafayette, USA) was transfected using Dharmafect reagent 2 (Dharmacon) according to the manufacturers protocol. Pools of 4 non-targeting siRNAs (cat\#D-001810-10-05) and PLK1 specific siRNAs (cat\#L-003290-00-0005) were used as negative and positive controls, respectively. The use of a non-targeting
siRNA pool ensures control for off-target effects. Transfection of cDNA encoding for myristoylated PIK3CA [13] Addgene, Cambridge, USA) and cotransfections with HPV16-URR luciferase constructs into FK16A cells were performed using Effectene (Qiagen, Hilden, Germany) according to instructions. Firefly luciferase and Renilla luciferase were measured using Dual Luciferase assay (Promega, Wisconsin, USA).

\section{Clinical material}

All tissue specimens were collected during the course of routine clinical practice at the Department of Obstetrics and Gynecology at the VU University medical center. Normal epithelial control samples were obtained from histologically normal frozen biopsies of non-cancer patients undergoing hysterectomy. This study followed the ethical guidelines of the Institutional Review Board of the VU University medical center.

\section{RNA isolation, RT-PCR and Northern Blotting}

Isolation of mRNA from cell lines was performed using RNA-B reagent (Tel-Test, Friendswood, USA) and DNase treated (Promega) prior to cDNA synthesis using specific reverse primers (see below). Total RNA from micro-dissected frozen biopsies of cervical SCCs and normal ectocervical controls was isolated using Trizol reagent (Invitrogen Life Technologies, Breda, The Netherlands) as described before [14]. Quantitative RT-PCR was performed as described previously [15] using the following primers for PIK3CA forward 5'-CCTGATCT TCCTCGTGCTGCTC-3' and reverse 5'- ATGCCAAT GGACAGTGTTCCTCTT -3' using SYBR Green PCR Master Mix (Applied Biosystems, Carlsbad, CA, USA). And for hTERT forward 5'-CACGCGAAAACCTTCCTCA -3', reverse 5'-CAAGTTCACCACGCAGCC-3' and the probe FAM-5'-CTCAGGGACACCTCGGACCAGGGT -3'-TAMRA using Universal PCR Master Mix (Applied Biosystems). To correct for RNA quality and input, we performed RT-PCR for the housekeeping gene snRNP as described before in cell line experiments [16]. For quantification, a standard curve was established using serial dilutions of cervical cancer cell line cDNA. To determine HPV16E7 mRNA expression LightCycler real-time PCR assays were applied as described before $[17,18]$ as well as Northern Blotting for HPV16. Total RNA was separated on a $1 \%$ agarose gel, blotted on nylon membranes (GeneScreen, PerkinElmer Life Sciences, Waltham, USA) and hybridized with a radioactive labelled full length HPV16 probe.

\section{Immunoblotting}

Antibodies against total (cat\#9272) or phosphorylated forms of PKB/AKT (cat\#4058), PIK3CA (cat\#4255) and loading control beta-actin (cat\#4967) (all 1:1000 from 
Cell Signaling Technology) were used according to the manufacturers instructions. Membranes were incubated with the appropriate horseradish peroxidase-conjugated secondary antibodies and the levels of corresponding proteins were visualized using SuperSignal West Dura Extended Duration Substrate (Pierce).

\section{Immunohistochemical staining and immunofluorescence assays}

Immunohistochemical staining was performed using 4 $\mu \mathrm{m}$ sections which were deparaffinised, rehydrated and microwave-treated $(800 \mathrm{~W})$ for $10 \mathrm{~min}$ in Tris buffer (pH9), followed by incubation for $30 \mathrm{~min}$ in $3 \% \mathrm{H}_{2} \mathrm{O}_{2}$ in methanol. Antibody incubation with PIK3CA (cat\#4249 Cell Signaling 1:200) was performed overnight at $4^{\circ} \mathrm{C}$ and for detection the EnVision horseradish peroxidase system (Dako, Heverlee, Belgium) was used.

For immunofluorescence, $4 \mu \mathrm{m}$ sections were rehydrated, treated with $10 \mathrm{mM}$ citrate, $\mathrm{pH} 6.0$ at $95^{\circ} \mathrm{C}$ for $10 \mathrm{~min}$ and allowed to cool to room temperature over 20 min. Slides were then treated with $3 \% \mathrm{H}_{2} \mathrm{O}_{2}$ in water and blocked in 1 XPBS containing $10 \%$ goat serum. P$\mathrm{PKB} / \mathrm{AKT}$ and Ki-67 were detected by sequential probing with respective antibodies because both were raised in rabbit. P-PKB/AKT was probed with rabbit monoclonal antibody (cat\#2118-1, Epitomics Inc, 1:100 dilution) and detected by fluorescine-conjugated tyramide (NEL701001, PerkinElmer Life Science, USA) as per the manufacturers direction. Subsequently, Ki-67 was probed with rabbit monoclonal antibody (ab16667, Abcam, 1:100 dilution) and detected by Alexa Fluor 555 conjugated anti-rabbit IgG (cat\#A21429, InvitrogenMolecular Probes, USA.) as per the manufacturers protocol. P-PKB/AKT and CK10 localization was detected by concurrent probing with respective primary antibodies as they were raised in different species. Raft sections were treated with rabbit anti p-PKB/AKT (described above) and mouse monoclonal anti-CK10 (cat\#ab1421, 1:150 dilution, Abcam, Cambridge, USA). Anti-CK10 was detected with Alexa Fluor 555 conjugated goat anti-mouse IgG (cat\#A21424, InvitrogenMolecular Probes, USA). Finally slides were mounted with DAPI containing media (VECTASHIELD, H1200, Vector Laboratories, USA), viewed under Olympus AX70 microscope fitted with Chroma filters. Photomicrographs were captured by Axiovision camera at $20 \times$ magnifications of objective and finally processed with Photoshop CS2 (Adobe) for documentation.

\section{Cell Viability, Migration, Anchorage Independent Growth}

Cell viability was assessed by MTT (3-(4,5-dimethylthiazol-2-yl)-2,5-diphenyltetrazolium bromide) dye reduction (ICN Biomedicals Inc, USA). Cells were seeded in triplicate wells in 96-wells plates, transfected or starved overnight followed by LY294002 treatment and grown for 5 days. Control conditions were set to $100 \%$.

For cellular migration assays cells were plated at high confluence and uniformly scratched to create a cell-free gap. After 24-48 hours in serum-free keratinocyte growth medium, plates were examined and photographed to asses the migration of neighbouring cells into the gap.

To examine anchorage independent growth under the different conditions 5000 cells of each condition were plated in semi-solid agarose (as described previously [19]). After 3 weeks colonies larger than $\sim 50$ cells were counted and pictures taken.

\section{Organotypic raft cultures}

The HPV containing keratinocytes were grown as epithelial raft tissues as described previously [20]. For all conditions duplicate rafts were developed. Transfections with siRNAs were carried out the day before seeding on the collagen beds. Inhibitor treatment was started after seeding and continued throughout the 9 days of culturing at the liquid-air interface. After harvesting, the raft tissues were fixed in formalin and embedded in paraffin. For histological examination, $4 \mu \mathrm{m}$ sections were stained with hematoxylin and eosin.

\section{Statistical analysis}

All statistical analyses were carried out using the T-test in the SPSS software package (SPSS 15.0, Chicago, USA).

\section{Results}

\section{Increased PIK3CA expression in cervical carcinomas}

To investigate the relevance of PI3-kinase pathway activation in cervical carcinogenesis, mRNA and protein expression levels of the catalytic subunit PIK3CA were examined. As can be seen in Figure 1A messenger RNA levels for PIK3CA were significantly higher in SCC ( $\mathrm{n}=$ 12) as compared to normal ectocervical controls ( $\mathrm{n}=$ 10) $(\mathrm{p}=0.005)$. All cervical carcinomas were previously analysed with arrayCGH and contained a gain of chromosome 3q [6], suggesting that elevated mRNA expression may be related to increased genomic content. A subset of cervical carcinomas was also analysed for PIK3CA protein expression using immunohistochemistry. Diffuse PIK3CA protein expression was observed in the tumor fields, whereas the upper strata were negative in adjacent normal cervical epithelium (Figure 1B).

\section{The PI3-kinase pathway becomes increasingly activated} during hrHPV-induced transformation in vitro

To examine whether the PI3-kinase pathway is relevant for HPV-mediated transformation in vitro, we made use of our model system consisting of primary keratinocytes 


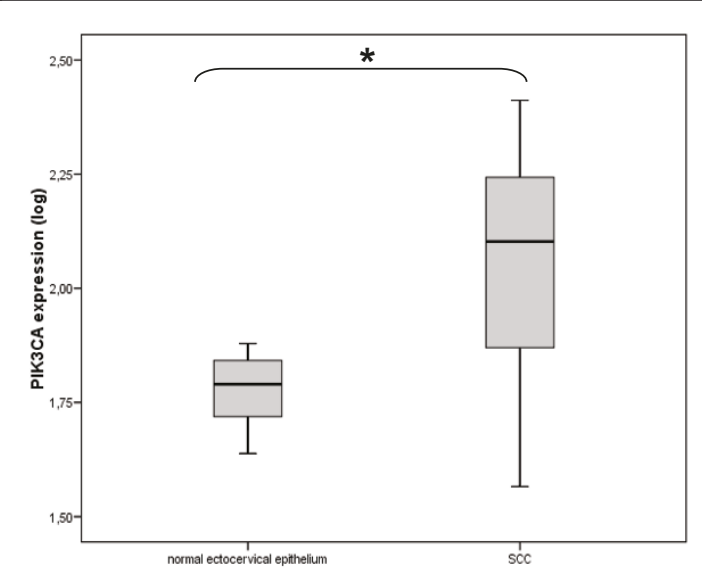

A
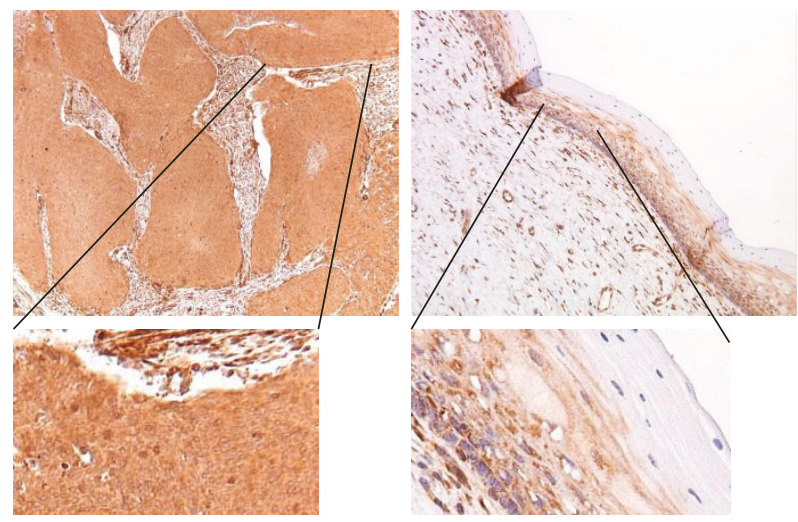

B

Figure 1 PIK3CA expression. (A) mRNA expression levels of PIK3CA in SCCs compared to normal ectocervical epithelium determined by quantitative RT-PCR. The upper and lower boundaries of the boxes represent the 75th to 25th percentile, bars within the boxes represent the median value. Whiskers represent the minimum and maximum values. (B) Representative pictures of immunostainings for PIK3CA on a cervical carcinoma specimen (left) and normal cervical epithelium (right) (20x magnification).

transfected with full-length HPV16. This longitudinal model consists of successive stages of transformation, including immortalization and anchorage-independent growth. The immortal cells have bypassed the senescence and crisis barriers that mark the end of the replicative and extended lifespan, respectively, and display increased telomerase activity [11]. At this stage, the cells also possess a gain of chromosome 3q [6]. Upon further passaging the immortal cells acquired an anchorageindependent phenotype [19].

HPV16-transfected keratinocytes (FK16A) representing these two different stages of transformation were analysed for PIK3CA mRNA and protein expression. One of the downstream targets of PIK3CA activation, the phosphorylated $\mathrm{PKB} / \mathrm{AKT}$, along with total $\mathrm{PKB} / \mathrm{AKT}$ were also analysed. FK16A cells showed an increase in PIK3CA mRNA expression with progression from immortal to an anchorage-independent phenotype (Figure 2A), which was associated with a slight elevation in PIK3CA protein expression (Figure 2B). During passaging a stronger increase in phosphorylation of PKB/AKT was seen (Figure 2B), indicative of a progressive activation PI3-kinase signalling. Analysis of HPV18 transfected keratinocytes derived from the same donor also revealed an increase in phosphorylated $\mathrm{PKB} / \mathrm{AKT}$ with passaging (Additional File 1, Figure S1). Similarly, HPV16E6E7 containing keratinocytes obtained from a second donor displayed increasing $\mathrm{p}-\mathrm{PKB} / \mathrm{AKT}$ levels with passaging (Additional File 2, Figure S2). The latter cells also showed a slight increase in PIK3CA protein expression at later passage. These data indicate that PI3kinase signalling becomes progressively activated during HPV-induced transformation and is donor and hrHPV type independent.
Phosphorylation and activation of $\mathrm{PKB} / \mathrm{AKT}$ is a result of upstream signalling events. Hence, we proceeded to determine whether we could influence PKB/AKT phosphorylation by modulating PI3-kinase activity in our model system. Different passages of FK16A cells were treated with the chemical PI3-kinase inhibitor LY294002, which fits in the ATP binding pocket of the catalytic subunit PIK3CA [21]. LY294002 treatment resulted in reduction of the levels of phosphorylated $\mathrm{PKB} / \mathrm{AKT}$ at all passages, while total $\mathrm{PKB} / \mathrm{AKT}$ remained the same (Figure $2 \mathrm{~B}$ ). These results indicate that phosphorylation of $\mathrm{PKB} / \mathrm{AKT}$ in these cells relies at least in part on the activity of PI3-kinase signalling.

\section{PI3-kinase signalling is functionally involved in hrHPV- induced transformation in vitro}

To examine the biological role of the PI3-kinase pathway in our cell lines, we determined the functional consequences of LY294002 treatment. Figure 3A illustrates that for both immortal and anchorage-independent passages of FK16A incubation with LY294002 resulted in a statistically significant reduction in the number of viable cells using a viability assay (MTT) $(\mathrm{p}=0.001$ for each passage). Another important feature of transformation is the migratory capacity of cells. Figure 3B shows that, upon wound induction, HPV16-tranformed keratinocytes migrated and closed the wound after 2 days. In contrast, chemical inhibition of PI3-kinase in these cells reduced their migratory capacity, as cells were unable to close the wound. Lastly, we examined anchorage-independent growth. At a concentration of $10 \mu \mathrm{M}$ of the inhibitor, colony formation was significantly inhibited compared to mock treated controls $(\mathrm{p}=0.003)$ and decreased further at a concentration of $20 \mu \mathrm{M}(\mathrm{p}=0.001)$ (Figure $3 \mathrm{C}$ and 


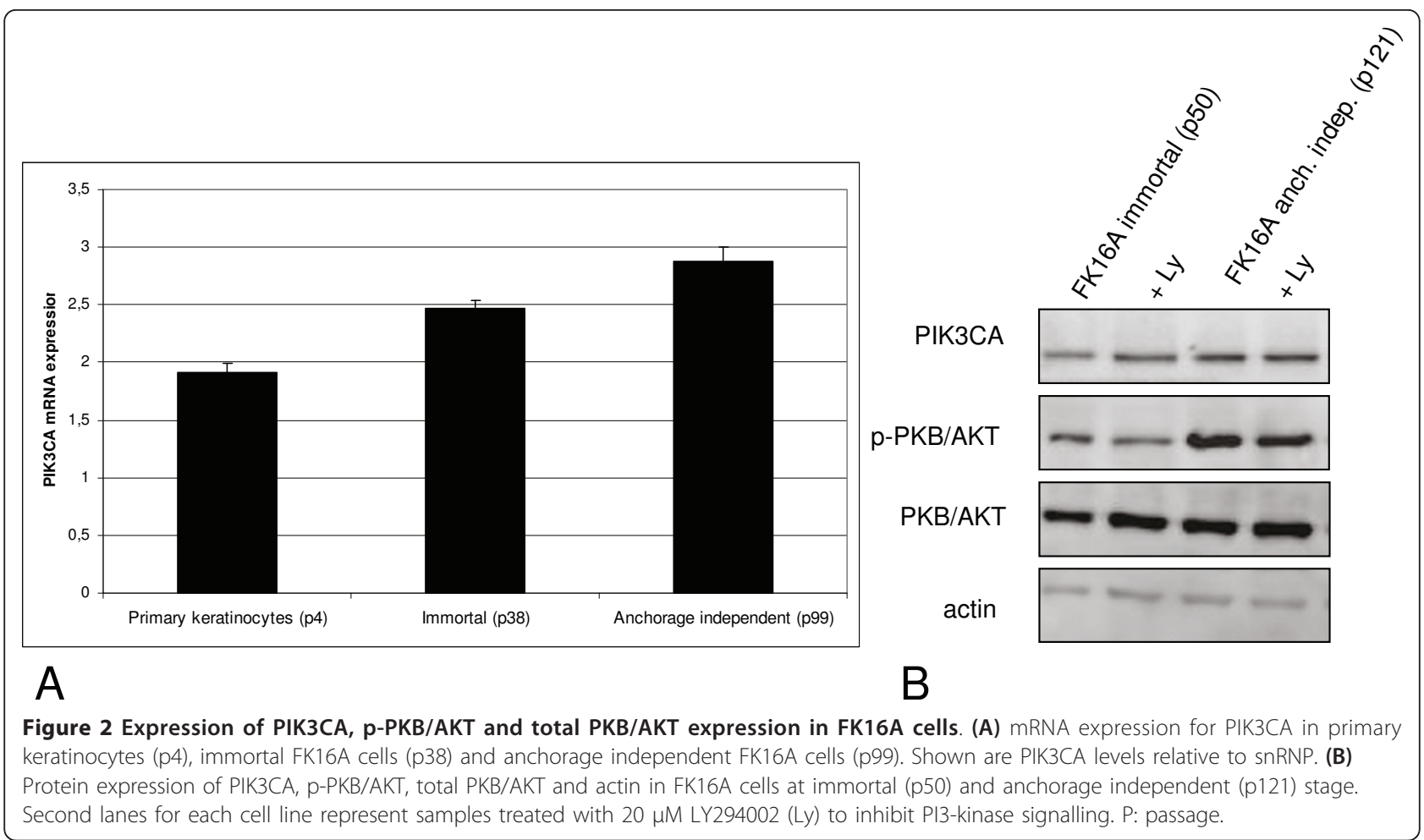

3D). Taken together, the results suggest that PI3-kinase signalling is functionally relevant for the transformed phenotypes of hrHPV-immortalized cells.

\section{The catalytic subunit PIK3CA is essential for HPV- mediated transformation}

Our findings strongly suggested a role of PIK3CA in HPV-mediated transformation. Since LY294002 is a universal PI3-kinase inhibitor affecting the different PI3kinase classes to varied extents, we next employed RNA interference to examine PIK3CA specifically. A pool of specific siRNAs was used to silence PIK3CA expression in anchorage independent FK16A cells. Non-targeting siRNAs were used to control for off-target effects. The reduction of PIK3CA expression was confirmed by Western blotting (Figure 4A). PIK3CA silencing strongly inhibited PI3-kinase signalling as reflected by reduced phosphorylation of $\mathrm{PKB} / \mathrm{AKT}$ without reduction of total $\mathrm{PKB} / \mathrm{AKT}$ (Figure 4A).

Following PIK3CA specific siRNA transfection cells were seeded for the functional tests described above. Similar to LY294002 treatment, siRNA-mediated PIK3CA knockdown significantly reduced the number of viable FK16A cells $(\mathrm{p}=0.0005)$ compared to transfection with a pool of non-targeting siRNAs (Figure 4B). Knockdown of PLK1, inducing cell death and used as a positive control, also resulted in a significant reduction of viable cells $(p=0.0003)$. FK16A cells transfected with siRNAs targeting PIK3CA also lost their migratory capacity and were unable to close the wound (Figure 4C). Primary human foreskin keratinocytes, included as controls, displayed a minor reduction in migratory capacity upon PIK3CA silencing (Additional File 3, Figure S3), in concordance with the lower level of PIK3CA mRNA in these cells (Figure 2A). Lastly, anchorage-independent growth was also affected by PIK3CA knockdown to a similar extent as after treatment with $10 \mu \mathrm{M}$ LY294002 (p = 0.05 ) (Figure 4D). Taken together, these results show that PIK3CA is relevant for the oncogenic properties of HPVmediated transformation.

To substantiate our hypothesis that PIK3CA is an important mediator of PI3-kinase signalling in HPV-transformed cells, we examined the effect of PIK3CA over-activation. This was achieved by transfection of anchorage independent FK16A cells with cDNA encoding myristoylated PIK3CA in which a coding sequence for the myristoylation domain of c-Src is fused in-frame with the PIK3CA coding sequence [13]. The amino-terminal myristoylation modification ensures membrane targeting of PIK3CA, allowing PIP3 production without the otherwise required receptor activation. Figure $5 \mathrm{~A}$ shows that transfection of PIK3CA cDNA resulted in increased levels of PIK3CA protein and also enhanced levels of $\mathrm{p}-\mathrm{PKB} / \mathrm{AKT}$. After treatment with LY294002, levels of p-PKB/AKT reverted, whereas levels of PIK3CA remained elevated. Viable cells increased significantly in FK16A cultures with ectopically activated PIK3CA when compared with untransfected cells $(\mathrm{p}=0.04)$. This effect was reversed by 


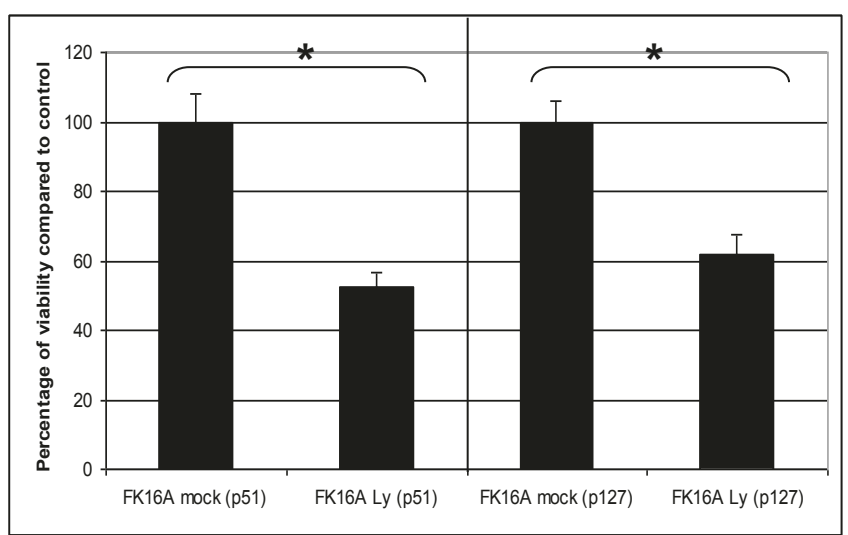

A

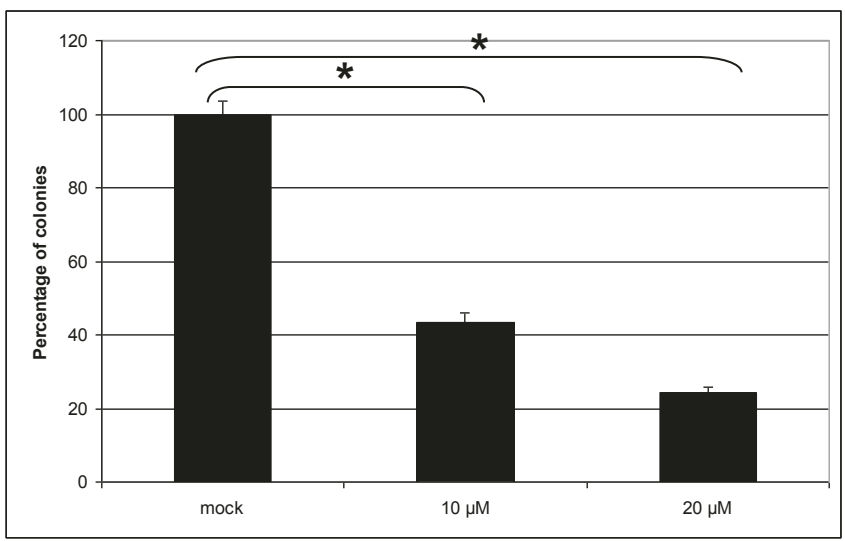

C

Anchorage independent cells ( $p 119)$

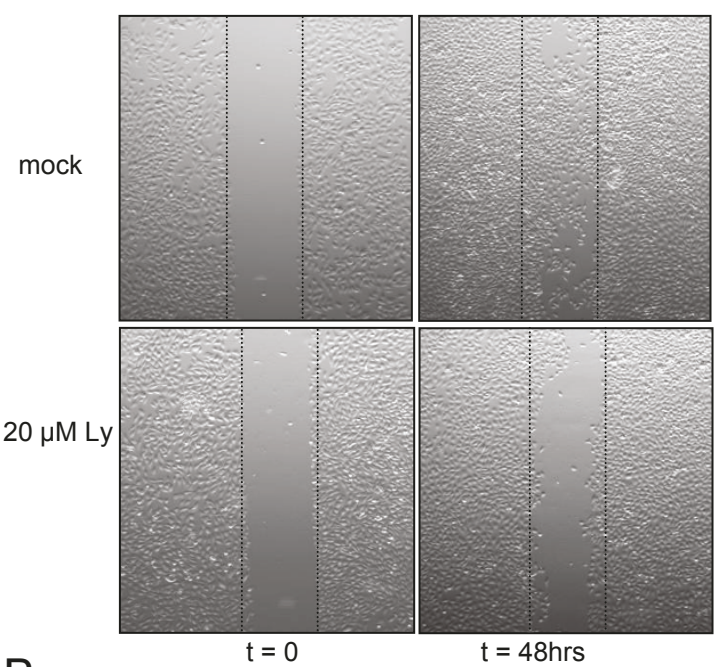

B

Anchorage independent cells (p123)

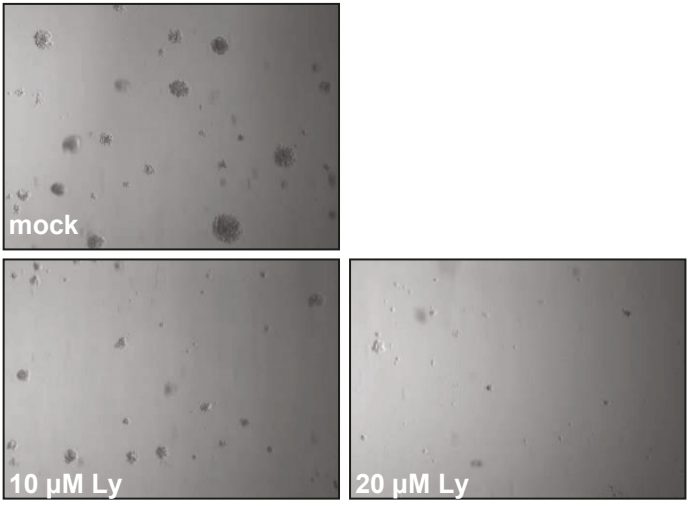

Anchorage independent cells ( $p 119)$

Figure 3 The functional consequences of silencing PI3-kinase signalling using the chemical inhibitor LY294002 (Ly) in FK16A cells. (A) Cell viability as measured 4 days post-treatment using an MTT assay. Appropriate controls were set to $100 \%$. Immortal (p51) and anchorage independent (p127) FK16A cells were treated with the PI3-kinase inhibitor LY294002. (B) Representative pictures of migration assay performed with anchorage independent FK16A cells (p123). Upper panel: mock treated cells, lower panel: cells treated with $20 \mu \mathrm{M}$ of the PI3-kinase inhibitor LY294002. Pictures were taken immediately after scratch induction and 48 hours post-induction. (C) Late passage FK16A cells (p119) were seeded in semi-solid agarose in the presence of the Pl3-kinase inhibitor. After 3 weeks in culture colonies were quantified relative to the appropriate controls, set to 100\%. * Indicates a statistical significant difference. (D) Representative pictures of anchorage independent growth results upon various LY294002 concentrations. P: passage.

incubation with $20 \mu \mathrm{M}$ LY294002 (Figure 5B). These findings further strengthen the notion of a role of PIK3CA and PI3-kinase signalling in HPV-containing cells.

\section{PI3-kinase signalling is involved in transcriptional regulation of $\mathrm{HPV}$}

In search of an explanation for the altered growth properties of the HPV16 containing keratinocytes upon manipulation of the PI3-kinase pathway, HPV16 expression was examined. Figure 6A shows that there was only a slight variation with increased passaging in HPV16 mRNA expression. After PI3-kinase inhibition in anchorage independent cells however, we found that HPV16 mRNA expression was strongly reduced as can be seen in the Northern Blot results in Figure 6B. Further quantification by real time RT-PCR revealed that HPV16E7 mRNA levels were significantly reduced after chemical inhibition to about half of the original expression at a concentration of $20 \mu \mathrm{M}$ LY294002 ( $\mathrm{p}=0.04$ ) (Figure 6C). To analyze further downstream effects of reduced HPV expression transcription of hTERT was monitored, which is known to be stimulated by E6 expression [22]. Cells in which PIK3CA was silenced and HPV mRNA expression was reduced also displayed a trend towards reduced hTERT 


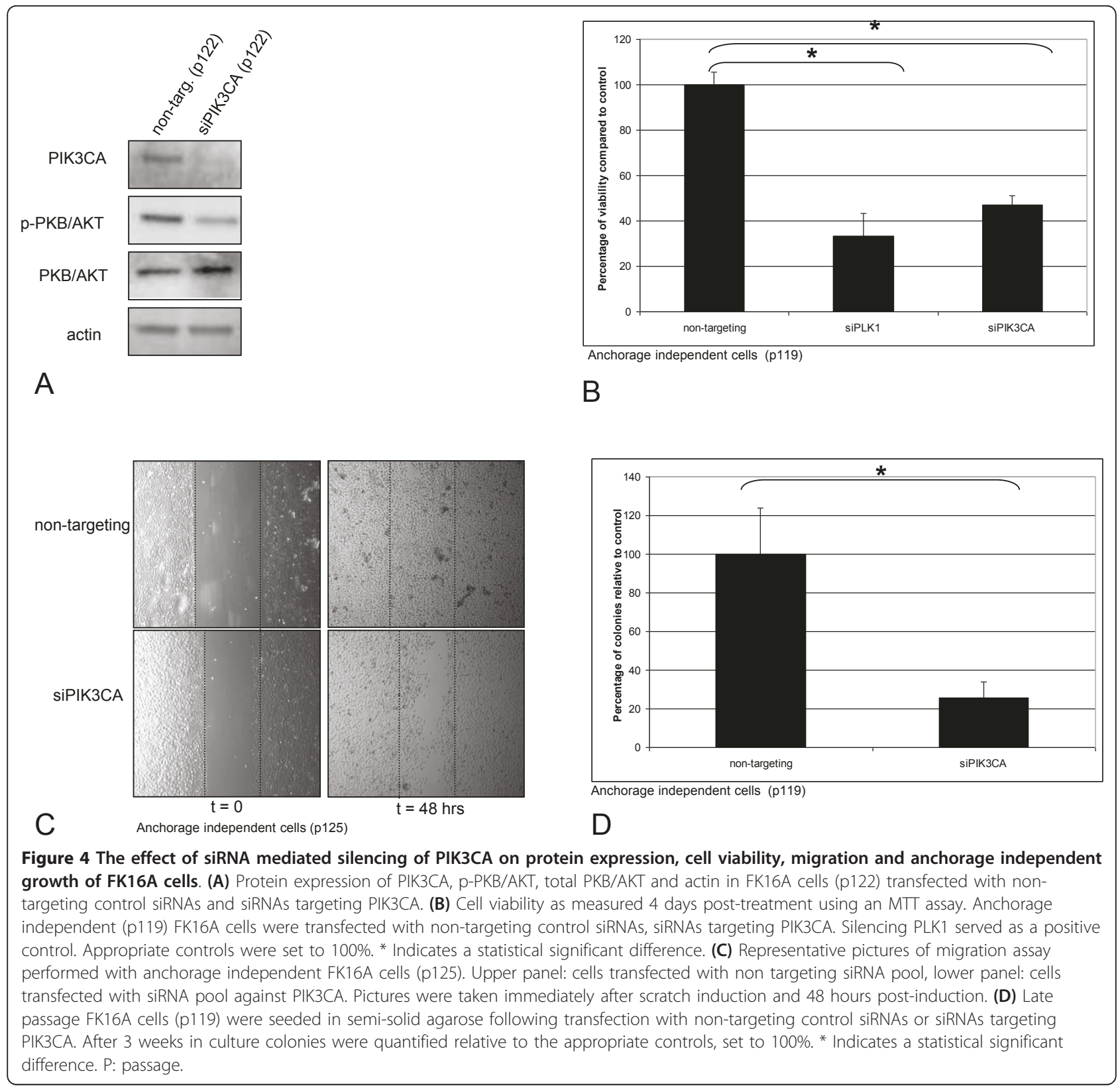

mRNA expression (Figure 6D). Although E7 mRNA expression levels in immortal and anchorage independent cells were comparable, while PIK3CA expression and PI3kinase activity were increased in anchorage independent cells, the results shown in Figure 6B-6D suggest a functional cross-talk between PI3-kinase and maintenance of $\mathrm{HPV}$ oncogene expression.

\section{PI3-kinase signalling is essential for growth and differentiation of HPV-immortalized cells in organotypic cultures}

Culturing human keratinocytes on organotypic raft mimics epithelial stratification and differentiation into various strata, whereby PI3-kinase signalling is known to play a role $[10,23-25]$. We have previously shown that differentiation is disturbed in HPV16- and HPV18immortalized keratinocytes, revealing phenotypes reminiscent of HPV-induced dysplastic lesions [20]. Thus, we next studied the roles of PI3-kinase signalling in growth and differentiation of these HPV-immortalized keratinocytes in organotypic raft cultures. FK16A cells displayed a severely dysplastic morphology on epithelial raft cultures, whereas an HPV18 immortalized cell line, FK18A, appeared less dysplastic and maintained terminal differentiation [20]. These different attributes permit a comparison of cell growth and differentiation upon 


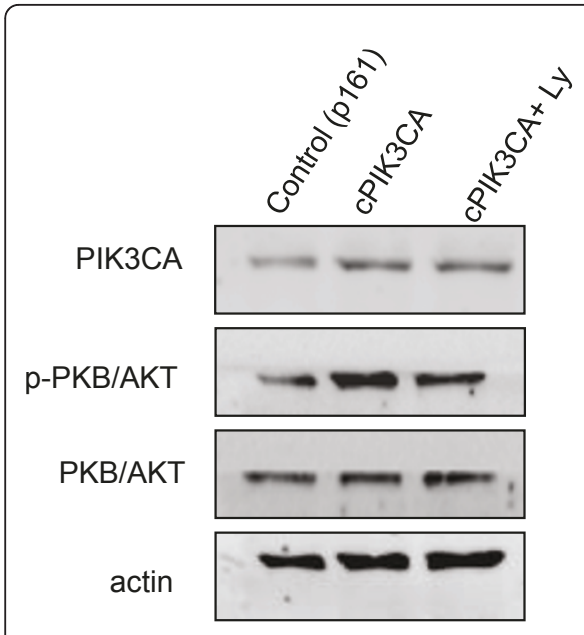

A



Anchorage independent cells ( $p 119)$

Figure 5 The effect of PIK3CA overexpression in FK16A cells. (A) Protein expression of PIK3CA, p-PKB/AKT, total PKB/AKT and actin in FK16A cells (p161) transfected with CDNA encoding PIK3CA as well as PIK3CA overexpressing cells treated with LY294002. (B) Cell viability as measured 4 days post-treatment using an MTT assay. Appropriate controls were set to $100 \%$ * Indicates a statistical significant difference.

modulating PI3-kinase. As described above, FK18A cells also exhibited increased levels of phosphorylated PKB/ AKT with passaging (Additional File 1, Figure S1).

Epithelial raft culturing of immortal FK16A cells in the presence of the PI3-kinase inhibitor or after silencing PIK3CA using siRNAs, hampered epithelial raft culture development compared to mock treated cells or cells treated with non-targeting siRNAs (Figure 7A). Particularly upon LY294002 treatment the epithelium was very thin, whereas transfection of PIK3CA-siRNAs had less effect on epithelial growth. These observations suggest that PIK3CA plays a role in the growth of these cells and that chemical inhibition of PI3-kinase most likely has additional effects besides $\mathrm{PKB} / \mathrm{AKT}$ inactivation. Similarly, raft culture growth of FK18A cells was strongly reduced upon chemical inhibition of PI3-kinase. No clear histological difference was seen in FK18A cells over-expressing activated PIK3CA (Figure 7A). Analysis of primary human foreskin keratinocytes showed no effect on growth on organotypic culture upon PIK3CA silencing (see Additional File 4, Figure S4).

To further assess the relation between phosphorylated $\mathrm{PKB} / \mathrm{AKT}$, cell proliferation and squamous differentiation, we performed double indirect immunostainings for p-PKB/AKT and Ki-67, a proliferation marker, or cytokeratin 10 (CK10), a differentiation marker.

As shown in Figure 7B, in raft cultures of FK16A cells, the $\mathrm{p}-\mathrm{PKB} / \mathrm{AKT}$ signals were primarily detected in the cytoplasm, while $\mathrm{Ki}-67$ was restricted to the nucleus. Both were detected stochastically throughout most of the epithelium. In the FK18A raft cultures, while Ki-67- positive cells were found in all cell strata, the p-PBK/ $\mathrm{AKT}$ signal was primarily detected in the upper most differentiated strata. Interestingly, the two signals rarely co-localized to the same cells.

After chemical inhibition of PI3-kinase, a fraction of the remaining epithelial cells were positive for Ki-67 despite the fact that phosphorylated PKB/AKT was strongly reduced or nearly abolished. In the presence of siRNAs targeting PIK3CA, the p-PKB/AKT signals in FK16A raft cultures were also greatly reduced while Ki67 signals were not altered. The non-targeting siRNAs had no effect on either. Upon ectopic expression of activated PIK3CA, more cells in the upper strata of the FK18A raft cultures were positive for $\mathrm{p}-\mathrm{PKB} / \mathrm{AKT}$ while the signals of $\mathrm{Ki}-67$ appeared unchanged.

Figure $7 \mathrm{C}$ shows that, in both cell lines, differentiation marker CK10 was positive from the suprabasal layers upwards. Consistent with the high dysplastic histology (Figure 7A), CK10 expression was patchy in the FK16A raft cultures with packets of CK10-negative cells, whereas the signals were much more uniformly detected across the FK18A cultures (Figure 7C). After chemical inhibition of PI3-kinase signalling in FK16A and FK18A cultures, not only $\mathrm{p}-\mathrm{PBK} / \mathrm{AKT}$ was largely abolished, but also the fraction of CK10-positve cells and signal strengths were both greatly reduced, especially in FK16A cultures. Similarly, in the siPIK3CA-treated FK16A cultures, p-PKB/AKT was virtually abrogated while CK10 signals were greatly reduced. In contrast, the non-targeting siRNAs had no effect on either signal. These results strongly suggest that differentiation was 


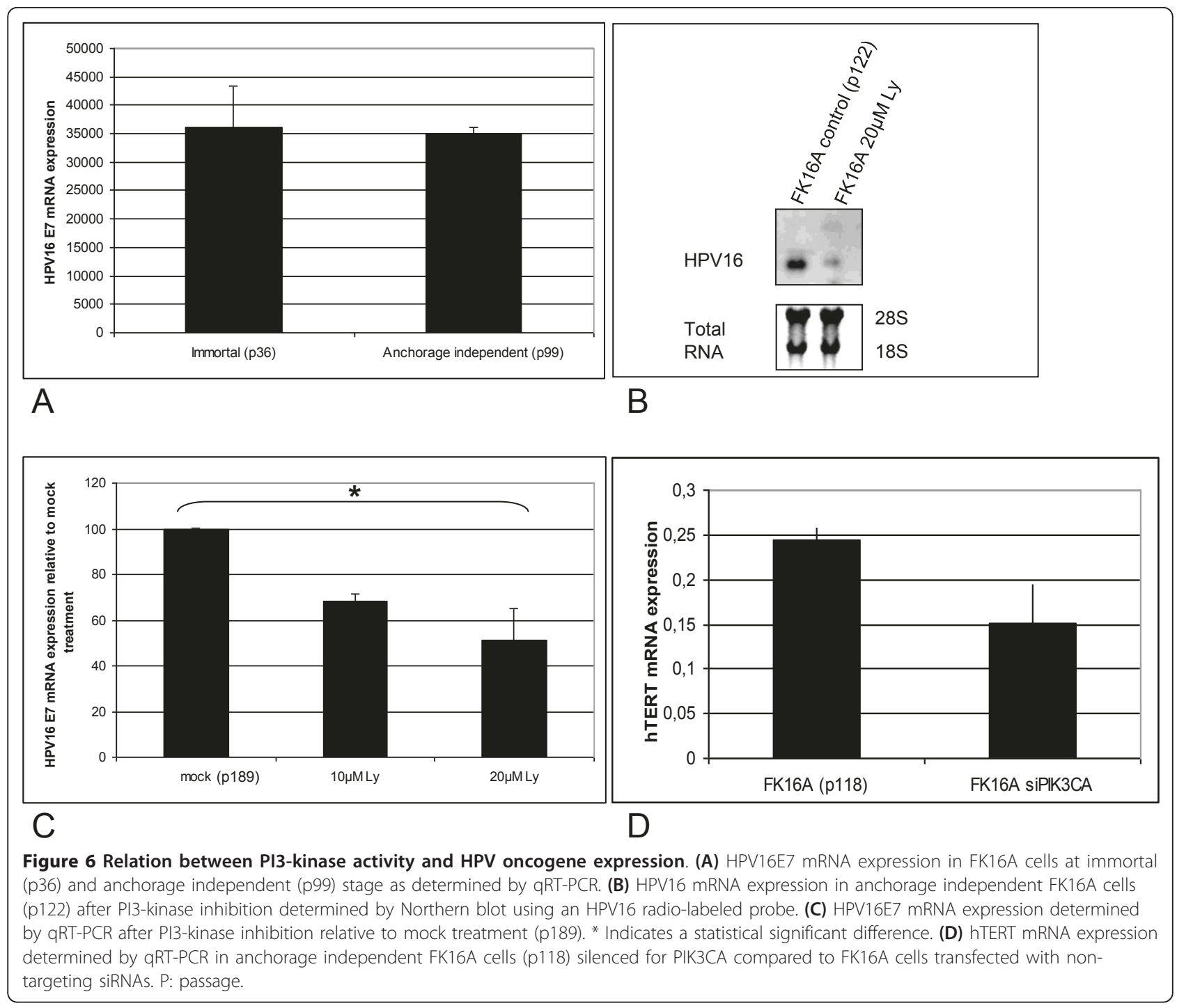

largely abolished upon reduction of $\mathrm{p}-\mathrm{PKB} / \mathrm{AKT}$. Despite an increased phophorylated PKB/AKT staining in FK18A cells overexpressing PIK3CA, CK10 staining patterns were similar to the non-transfected controls, because these cells were already strongly positive for CK10. These data confirm that PI3-kinase signalling is important for regulating differentiation of HPV-immortalized cells.

\section{Discussion}

The PI3-kinase/PKB/AKT signalling pathway affects a wide variety of cellular characteristics such as proliferation, differentiation and cell survival and is often altered in many human malignancies [7]. In cervical cancer, a gain of the long arm of chromosome 3, where PIK3CA is located, is often described and suggested to be a compulsory second hit for malignant transformation following an hrHPV infection $[4,6]$. However, data is scarce on the biological effects of altered PIK3CA expression and PI3-kinase signalling in cervical carcinogenesis.

In the present study we showed that mRNA expression of the catalytic subunit PIK3CA was significantly upregulated in cervical SCC. In our previous studies using arrayCGH, up to $100 \%$ of cervical SCC were shown to contain additional copies of chromosome 3q [6]. Additionally, low levels PIK3CA amplifications were found in $40 \%$ $74 \%$ of cervical carcinomas [5,8,26]. Bertelsen et al showed that an increase in PIK3CA copy numbers (more than 3 copies) was significantly correlated to elevated p-PKB/ AKT expression in cervical SCC and high-grade precursor lesions [8]. Also, other studies reported an increase in p$\mathrm{PKB} / \mathrm{AKT}$ staining with increase of histopathological grade of cervical disease $[9,10]$.

Functionally, we showed that PIK3CA and concomitant PI3-kinase signalling is involved in the different stages of HPV-mediated transformation in vitro. 


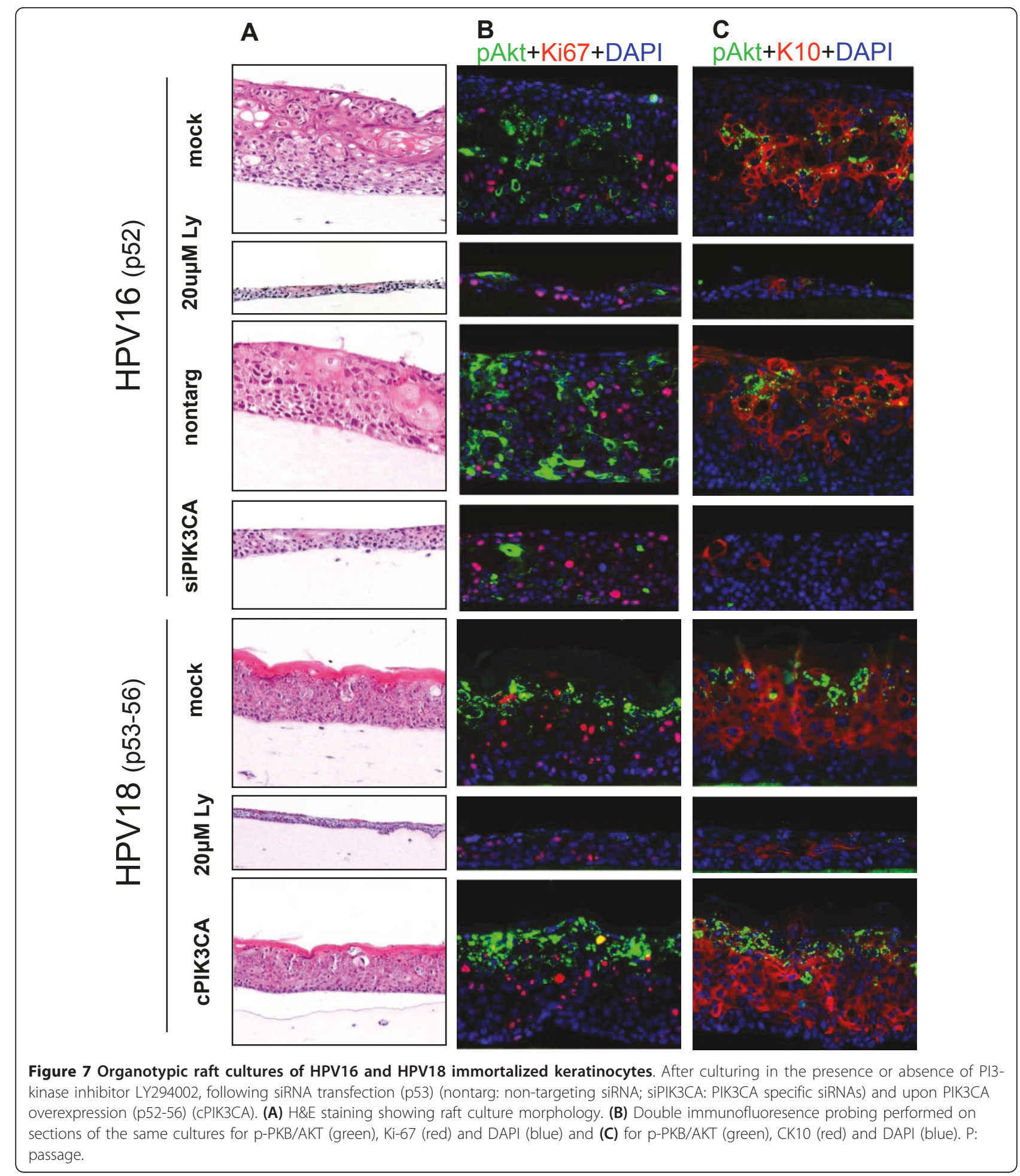

Modulating PI3-kinase activity in HPV transfected keratinocytes affected proliferation, migration, anchorage independent growth, and epithelial growth and differentiation in organotypic cultures. These data are consistent with previous reports using different cellular systems.
For instance, in ovarian cancer cell lines PI3-kinase pathway inhibition with LY294002 resulted in reduced proliferation via cell cycle arrest in G1 [27] or induction of apoptosis [28]. Proliferation measured by cell number and $\left[{ }^{3} \mathrm{H}\right]$-Thymidine incorporation was also reduced in 
rat intestinal epithelial cells as a result of LY294002 treatment [29]. Furthermore, the involvement of PI3kinase in colony formation has been shown in mammary epithelial cells where overexpression of PIK3CA increased colony formation while a dominant negative form of PIK3R1 (p85) lacking the PIK3CA binding domain repressed colony formation [30]. This latter study also showed that, although $\mathrm{PKB} / \mathrm{AKT}$ is the main effector of PI3-kinase, PKB/AKT activation cannot substitute PI3-kinase signalling.

Functional studies in cervical cells are restricted to the common hrHPV positive cervical cancer cell lines such as $\mathrm{SiHa}, \mathrm{HeLa}$ and CaSki and did not include the explicit analysis of PIK3CA as a candidate oncogene. Treatment of SiHa with the PI3-kinase inhibitor LY294002 led to reduced proliferation and increased apoptotic DNA fragments [5]. For HeLa and CaSki the reduction in proliferation upon LY294002 treatment was shown to be independent of apoptosis, though did sensitize the cells to radiation [31].

None of the previous studies on cancer cells have had the benefit of a longitudinal characterization as is afforded by our in vitro model system, which mimics the different stages of cervical carcinogenesis. We were able to show a progressive upregulation of PI3-kinase signalling during HPV-induced transformation by using the elevation of $\mathrm{p}-\mathrm{PKB} / \mathrm{AKT}$ as a reporter. Indeed, $\mathrm{p}-$ $\mathrm{PBK} / \mathrm{AKT}$ was elevated in anchorage independent cells relative to HPV-immortalized cells. The expression levels of both PIK3CA and especially p-PKB/AKT in the latter cells were increased compared to primary keratinocytes (data not shown). This increased signalling functionally correlated with multiple attributes of transformed cells, such as cell growth, migration, and anchorage independent growth (Figure 3 ). The fact that specific silencing of PIK3CA using RNA interference affected each of the same transformed phenotypes in a negative manner, while PIK3CA overexpression increased proliferation (Figures 4 and 5) substantiates its function as an oncogene in cervical carcinogenesis, as has been suggested in previous studies $[5,8,26]$.

Remarkably, our data also suggest a feedback effect in which PI3-kinase regulates HPV oncogene expression. HPV16 mRNA expression and hTERT mRNA were decreased after inhibition of PI3-kinase signalling (Figure 6). The downregulation of hTERT mRNA may be a direct result of reduced HPV expression, as E6 activates hTERT transcription [22]. Thus, the reciprocal regulation between PIK3CA/PI3-kinase and viral oncogene expression acts in concert in maintaining the transformed phenotypes [32-34]. However, it is presently unknown whether the phenotypical effects of PI3-kinase inhibition seen in our model system reflect a direct consequence of E6/E7 repression or vice versa.
It has been shown that the oncoprotein E7 is able to activate $\mathrm{PKB} / \mathrm{AKT}$, which appeared to be dependent on its ability to bind and inactivate $\mathrm{Rb}$ gene family proteins $[10,35]$. E7 may also maintain PKB/AKT in an active state, by binding and sequestering a known binding partner, the phosphatase PP2A, thereby inhibiting dephosphorylation of p-PKB/AKT [36]. Active PKB/ AKT is also known to activate MDM2, enhancing p53 degradation and elevating $\mathrm{CDKs}$ leading to $\mathrm{pRb}$ inactivation, further enhancing the established E6/E7 effect on p53 and pRb function [37,38]. Moreover, activation of PKB/AKT by PI3-kinase can inhibit nuclear localization of p27kip1 and p21cip by phosphorylating the nuclear localization signal and hereby preventing nuclear transport and inducing proliferation, both of which are also affected by E7 overexpression [35,39]. Here we show that besides the reported effect of HPVE7 on PI3-kinase activity, there is also a feed-back effect in that PI3kinase can regulate HPV oncogene expression. From the present data it becomes clear that both mechanisms act in concert as the increased PI3-kinase activity appears essential for maintaining HPV oncogene expression. The need for a strict regulation of HPV expression, as also implicated in our previous study [40], suggests that yet other transformation-inducing host cell alterations contribute to the regulated expression of HPVE6/E7 as well as PI3-kinase signalling activity.

Organotypic raft cultures mimic a natural environment to evaluate the role of PI3-kinase signalling in the growth and differentiation of keratinocytes. Phosphorylation of $\mathrm{PKB} / \mathrm{AKT}$ appeared to be tightly linked to differentiation, which is in agreement with a previous report showing upregulation of phosphorylated PKB/ AKT in organotypic raft cultures of HPV16 expressing keratinocytes [10]. Inhibiting PI3-kinase signalling by chemical inhibition and PIK3CA siRNA-mediated silencing during raft formation led to a dramatic reduction in $\mathrm{p}-\mathrm{PKB} / \mathrm{AKT}$ and severely hampered epithelial cell growth in both HPV16- and HPV18 immortalized cells (Figure 7). Proliferation marker Ki-67 remained detectable in residual epithelial cells, while differentiation marker CK10 was dramatically reduced. These results indicate that PIK3CA regulated p-PKB/AKT expression is important in squamous differentiation. This conclusion is in accordance with a previous report showing that mouse keratinocytes with active PKB/AKT have higher levels of differentiation markers Keratin 1, filaggrin and loricrin. Moreover, inhibition of PI3-kinase resulted in the specific death of differentiating keratinocytes [23]. Similarly, treatment of primary esophageal keratinocytes with LY294002 resulted in an overall decrease in the number of basal keratinocytes and thickness of the epithelium [41]. It has been suggested that PI3-kinase becomes important upon commitment of 
keratinocytes to differentiation, initiating the process and subsequently delivering the required survival signals [24]. Based on the histology of our raft cultures, there were no apoptotic cells that are typified by highly condense and shrunken nuclei (Figure 7A). The thinning of the epithelium appears to stem from a severely curtailed proliferation. Hence, in organotypic raft culture systems, p-PKB/AKT appears to correlate with squamous differentiation and survival but not with proliferation, as is also evident from the lack of co-localization between p$\mathrm{PKB} / \mathrm{AKT}$ and Ki-67 (Figure 7). Also in a study by Menges et al [10] p-AKT is seen in differentiated nonproliferating BrdU negative cells. These observation are also in line with previous studies showing that AKT knock-out MEFs reduce BrdU incorporation by only 44$61 \%$ [42], indicating that AKT is not indispensible for proliferation, though the lack of it would slow down cell cycle progression at G1-S. We hypothesize that the balance in the dual character of PI3-kinase/PKB/AKT signalling shifts during the process of HPV-induced transformation towards tumor characteristics, rather than differentiation.

\section{Conclusions}

We have demonstrated that activation of the PI3-kinase/ $\mathrm{PKB} / \mathrm{AKT}$ pathway, in part resulting from increased PIK3CA expression, is functionally involved in HPVmediated transformation in vitro. PIK3CA-mediated PI3-kinase signalling appeared essential in regulating proliferation, anchorage-independent growth, migration and importantly viral oncogene expression required to maintain the transformed phenotypes. Moreover, PIK3CA expression and PI3-kinase signalling emerged as critical regulators of epithelial growth and differentiation in organotypic raft cultures of HPV-immortalized cells. Hence, PIK3CA and/or the PI3-kinase/PKB/AKT pathway may provide suitable targets for therapeutic intervention in patients with HPV-induced carcinomas.

\section{Additional material}

Additional file 1: Figure S1. Phosporylated-PKB/AKT and total PKB/ AKT protein expression in HPV18-containing keratinocytes. Protein lysates of HPV18-containing keratinocytes at passage 31 and passage 95 were analysed for p-PKB/AKT, PKB/AKT and actin protein expression by Western blot analysis.

Additional file 2: Figure S2. PIK3CA, phosporylated-PKB/AKT and total PKB/AKT protein expression in HPV16E6E7 containing keratinocytes from a different donor. Lysates were analysed for protein expression at passage 13 and passage 60 for PIK3CA, p-PKB/AKT, PKB/AKT and actin.

Additional file 3: Figure S3. Migration assay of primary human foreskin keratinocytes without and with PIK3CA silencing. Representative pictures of migration assay performed with primary keratinocytes (p5). Upper panel: cells transfected with non-targeting siRNA pool, lower panel: cells transfected with siRNA pool against
PIK3CA. Pictures were taken immediately after scratch induction and 24 hours post-induction.

Additional file 4: Figure S4. Organotypic raft cultures of primary human foreskin keratinocytes without and with PIK3CA silencing. H\&E staining showing raft culture morphology following transfection of primary human foreskin keratinocytes (p3) with non-targeting siRNAs (left) and siRNAs targeting PIK3CA (right).

\section{Acknowledgements}

This research was supported by the VUmc Institute of Cancer and Immunology (V-ICI) and by USPHS grant CA83679 to LTC. We thank W Vos, D Claassen-Kramer, DM Schütze, L Bosch, and SM Wilting for excellent technical assistance and TM Roberts for providing the PIK3CA construct. We also thank AE Greijer and DAM Heideman for helpful discussion.

\section{Author details}

${ }^{1}$ Department of Pathology, Unit of Molecular Pathology, VU University Medical Center, PO Box 7057, 1007 MB Amsterdam, The Netherlands. ${ }^{2}$ Department of Biochemistry and Molecular Genetics, University of Alabama at Birmingham, Birmingham, Alabama 35294-0005, USA. ${ }^{3}$ Division of Viral Transformation Mechanisms, German Cancer Center, Im Neuenheimer Feld 242,D-69120 Heidelberg Germany.

\section{Authors' contributions}

FEH performed experiments and drafted the manuscript. NSB carried out the immunofluorescent stainings. RDMS, PJFS, CJLMM, LTC, TRB participated in design of the study and interpretation of data and helped to draft the manuscript with critical revisions for intellectual content. JDCA and FR participated in HPV expression analysis and critically revised the manuscript. All authors read and approved the final manuscript.

\section{Competing interests}

The authors Prof dr LT Chow, Prof dr T Broker and dr NS Banerjee are funded by $\mathrm{NIH}$. Other authors have no competing interests.

Received: 5 November 2010 Accepted: 10 June 2011

Published: 10 June 2011

\section{References}

1. zur Hausen $\mathrm{H}$ : Papillomavirus infections-a major cause of human cancers. Biochim Biophys Acta 1996, 1288:F55-F78.

2. von Knebel-Doeberitz M, Oltersdorf T, Schwarz E, Gissmann L: Correlation of modified human papilloma virus early gene expression with altered growth properties in C4-1 cervical carcinoma cells. Cancer Res 1988, 48:3780-3786.

3. von Knebel-Doeberitz M, Rittmuller $\mathrm{C}$, zur HH, Durst M: Inhibition of tumorigenicity of cervical cancer cells in nude mice by HPV E6-E7 antisense RNA. Int J Cancer 1992, 51:831-834.

4. Heselmeyer K, Macville M, Schrock E, Blegen H, Hellstrom AC, Shah K, Auer $G$, Ried T: Advanced-stage cervical carcinomas are defined by a recurrent pattern of chromosomal aberrations revealing high genetic instability and a consistent gain of chromosome arm 3q. Genes Chromosomes Cancer 1997, 19:233-240.

5. Ma YY, Wei SJ, Lin YC, Lung JC, Chang TC, Whang-Peng J, Liu JM, Yang DM, Yang WK, Shen CY: PIK3CA as an oncogene in cervical cancer. Oncogene 2000, 19:2739-2744.

6. Wilting SM, Snijders PJ, Meijer GA, Ylstra B, van den ljssel PR, Snijders AM, Albertson DG, Coffa J, Schouten JP, van de Wiel MA, et al: Increased gene copy numbers at chromosome $20 \mathrm{q}$ are frequent in both squamous cell carcinomas and adenocarcinomas of the cervix. J Pathol 2006, 209:220-230

7. Nicholson KM, Anderson NG: The protein kinase B/Akt signaling pathway in human malignancy. Cell Signal 2002, 14:381-395.

8. Bertelsen BI, Steine SJ, Sandvei R, Molven A, Laerum OD: Molecular analysis of the PI3K-AKT pathway in uterine cervical neoplasia: frequent PIK3CA amplification and AKT phosphorylation. Int J Cancer 2006, 118:1877-1883.

9. Kohrenhagen N, Voelker HU, Schmidt M, Kapp M, Krockenberger M, Frambach T, Dietl J, Kammerer U: Expression of transketolase-like 1 
(TKTL1) and p-Akt correlates with the progression of cervical neoplasia. $J$ Obstet Gynaecol Res 2008, 34:293-300.

10. Menges CW, Baglia LA, Lapoint R, McCance DJ: Human papillomavirus type $16 \mathrm{E7}$ up-regulates AKT activity through the retinoblastoma protein. Cancer Res 2006, 66:5555-5559.

11. Steenbergen RD, Walboomers JM, Meijer CJ, van der Raaij-Helmer EM, Parker JN, Chow LT, Broker TR, Snijders PJ: Transition of human papillomavirus type 16 and 18 transfected human foreskin keratinocytes towards immortality: activation of telomerase and allele losses at $3 p$, 10p, 11q and/or 18q. Oncogene 1996, 19(13):1249-1257.

12. Struijk L, van der ME, Kazem S, ter SJ, de Gruijl FR, Steenbergen RD, Feltkamp MC: Specific betapapillomaviruses associated with squamous cell carcinoma of the skin inhibit UVB-induced apoptosis of primary human keratinocytes. J Gen Virol 2008, 89:2303-2314.

13. Zhao JJ, Liu Z, Wang L, Shin E, Loda MF, Roberts TM: The oncogenic properties of mutant p110alpha and p110beta phosphatidylinositol 3kinases in human mammary epithelial cells. Proc Natl Acad Sci USA 2005, 102:18443-18448.

14. Wilting SM, de Wilde J, Meijer CJ, Berkhof J, Yi Y, van Wieringen WN, Braakhuis BJ, Meijer GA, Ylstra B, Snijders PJ, et al: Integrated genomic and transcriptional profiling identifies chromosomal loci with altered gene expression in cervical cancer. Genes Chromosomes Cancer 2008, 47:890-905.

15. de Wilde J, Wilting SM, Meijer CJ, van de Wiel MA, Ylstra B, Snijders PJ, Steenbergen RD: Gene expression profiling to identify markers associated with deregulated hTERT in HPV-transformed keratinocytes and cervical cancer. Int J Cancer 2008, 122:877-888.

16. de Wilde J, De-Castro Arce J, Snijders PJ, Meijer CJ, Rosl F, Steenbergen RD: Alterations in AP-1 and AP-1 regulatory genes during HPV-induced carcinogenesis. Cell Oncol 2008, 30:77-87.

17. Hesselink AT, van den Brule AJ, Groothuismink ZM, Molano M, Berkhof J, Meijer CJ, Snijders PJ: Comparison of three different PCR methods for quantifying human papillomavirus type 16 DNA in cervical scrape specimens. J Clin Microbiol 2005, 43:4868-4871.

18. Snijders PJ, Hogewoning CJ, Hesselink AT, Berkhof J, Voorhorst FJ, Bleeker MC, Meijer CJ: Determination of viral load thresholds in cervical scrapings to rule out CIN 3 in HPV 16, 18, 31 and 33-positive women with normal cytology. Int J Cancer 2006, 119:1102-1107.

19. Steenbergen RD, Kramer D, Braakhuis BJ, Stern PL, Verheijen RH, Meijer CJ, Snijders PJ: TSLC1 gene silencing in cervical cancer cell lines and cervical neoplasia. J Natl Cancer Inst 2004, 96:294-305.

20. Steenbergen RD, Parker JN, Isern S, Snijders PJ, Walboomers JM, Meijer CJ, Broker TR, Chow LT: Viral E6-E7 transcription in the basal layer of organotypic cultures without apparent p21cip1 protein precedes immortalization of human papillomavirus type 16- and 18-transfected human keratinocytes. J Virol 1998, 72:749-757.

21. Vlahos CJ, Matter WF, Hui KY, Brown RF: A specific inhibitor of phosphatidylinositol 3-kinase, 2-(4-morpholinyl)-8-phenyl-4H-1benzopyran-4-one (LY294002). J Biol Chem 1994, 269:5241-5248.

22. Klingelhutz AJ, Foster SA, McDougall JK: Telomerase activation by the E6 gene product of human papillomavirus type 16. Nature 1996, 380:79-82

23. Calautti E, Li J, Saoncella S, Brissette JL, Goetinck PF: Phosphoinositide 3kinase signaling to Akt promotes keratinocyte differentiation versus death. J Biol Chem 2005, 280:32856-32865.

24. Janes SM, Ofstad TA, Campbell DH, Eddaoudi A, Warnes G, Davies D, Watt FM: PI3-kinase-dependent activation of apoptotic machinery occurs on commitment of epidermal keratinocytes to terminal differentiation. Cell Res 2009, 19:328-339.

25. Segrelles C, Moral M, Lara MF, Ruiz S, Santos M, Leis H, Garcia-Escudero R, Martinez-Cruz AB, Martinez-Palacio J, Hernandez P, et al: Molecular determinants of Akt-induced keratinocyte transformation. Oncogene 2006, 25:1174-1185.

26. Zhang A, Maner S, Betz R, Angstrom T, Stendahl U, Bergman F, Zetterberg A, Wallin KL: Genetic alterations in cervical carcinomas: frequent low-level amplifications of oncogenes are associated with human papillomavirus infection. Int I Cancer 2002, 101:427-433.

27. Gao N, Flynn DC, Zhang Z, Zhong XS, Walker V, Liu KJ, Shi X, Jiang BH: G1 cell cycle progression and the expression of $\mathrm{G} 1$ cyclins are regulated by $\mathrm{PI3K} / \mathrm{AKT} / \mathrm{mTOR} / \mathrm{p} 70 \mathrm{~S} 6 \mathrm{~K} 1$ signaling in human ovarian cancer cells. Am J Physiol Cell Physiol 2004, 287:C281-C291.
28. Shayesteh L, Lu Y, Kuo WL, Baldocchi R, Godfrey T, Collins C, Pinkel D, Powell B, Mills GB, Gray JW: PIK3CA is implicated as an oncogene in ovarian cancer. Nat Genet 1999, 21:99-102.

29. Sheng H, Shao J, Townsend CM, Evers BM: Phosphatidylinositol 3-kinase mediates proliferative signals in intestinal epithelial cells. Gut 2003, 52:1472-1478,

30. Zhao JJ, Gjoerup OV, Subramanian RR, Cheng Y, Chen W, Roberts TM, Hahn WC: Human mammary epithelial cell transformation through the activation of phosphatidylinositol 3-kinase. Cancer Cell 2003, 3:483-495.

31. Lee CM, Fuhrman CB, Planelles V, Peltier MR, Gaffney DK, Soisson AP, Dodson MK, Tolley HD, Green CL, Zempolich KA: Phosphatidylinositol 3kinase inhibition by LY294002 radiosensitizes human cervical cancer cell lines. Clin Cancer Res 2006, 12:250-256.

32. Defilippis RA, Goodwin EC, Wu L, DiMaio D: Endogenous human papillomavirus E6 and E7 proteins differentially regulate proliferation, senescence, and apoptosis in HeLa cervical carcinoma cells. J Virol 2003, 77:1551-1563.

33. Wells SI, Francis DA, Karpova AY, Dowhanick JJ, Benson JD, Howley PM: Papillomavirus E2 induces senescence in HPV-positive cells via pRB- and p21(CIP)-dependent pathways. EMBO J 2000, 19:5762-5771.

34. Wells SI, Aronow BJ, Wise TM, Williams SS, Couget JA, Howley PM: Transcriptome signature of irreversible senescence in human papillomavirus-positive cervical cancer cells. Proc Natl Acad Sci USA 2003, 100:7093-7098.

35. Charette ST, McCance DJ: The E7 protein from human papillomavirus type 16 enhances keratinocyte migration in an Akt-dependent manner. Oncogene 2007, 26:7386-7390.

36. Pim D, Massimi P, Dilworth SM, Banks L: Activation of the protein kinase B pathway by the HPV-16 E7 oncoprotein occurs through a mechanism involving interaction with PP2A. Oncogene 2005, 24:7830-7838.

37. Liang J, Slingerland JM: Multiple roles of the PI3K/PKB (Akt) pathway in cell cycle progression. Cell Cycle 2003, 2:339-345.

38. Zhou BP, Liao Y, Xia W, Zou Y, Spohn B, Hung MC: HER-2/neu induces p53 ubiquitination via Akt-mediated MDM2 phosphorylation. Nat Cell Biol 2001, 3:973-982.

39. Westbrook TF, Nguyen DX, Thrash BR, McCance DJ: E7 abolishes rafinduced arrest via mislocalization of p21(Cip1). Mol Cell Biol 2002, 22:7041-7052.

40. Van Tine BA, Kappes JC, Banerjee NS, Knops J, Lai L, Steenbergen RD, Meijer CL, Snijders PJ, Chatis P, Broker TR, et al: Clonal selection for transcriptionally active viral oncogenes during progression to cancer. J Virol 2004, 78:11172-11186.

41. Oyama K, Okawa T, Nakagawa H, Takaoka M, Andl CD, Kim SH, KleinSzanto A, Diehl JA, Herlyn M, El-Deiry W, et al: AKT induces senescence in primary esophageal epithelial cells but is permissive for differentiation as revealed in organotypic culture. Oncogene 2007, 26:2353-2364.

42. Skeen JE, Bhaskar PT, Chen CC, Chen WS, Peng XD, Nogueira V, HahnWindgassen A, Kiyokawa H, Hay N: Akt deficiency impairs normal cell proliferation and suppresses oncogenesis in a p53-independent and mTORC1-dependent manner. Cancer Cell 2006, 10:269-280.

\section{doi:10.1186/1476-4598-10-71}

Cite this article as: Henken et al:: PIK3CA-mediated PI3-kinase signalling is essential for HPV-induced transformation in vitro. Molecular Cancer 2011 10:71.

\section{Submit your next manuscript to BioMed Central and take full advantage of:}

- Convenient online submission

- Thorough peer review

- No space constraints or color figure charges

- Immediate publication on acceptance

- Inclusion in PubMed, CAS, Scopus and Google Scholar

- Research which is freely available for redistribution 